\title{
Impaired Spatial Learning and Reduced MK-801 Associated Behavioral Deficits in Rodents Following Early Postnatal Exposure to Low-level Lead
}

\author{
Jing Guo \\ Department of Life Sciences \\ University of Toronto \\ 1265 Military Trail \\ Toronto, Ontario M1C 1A4 CANADA
}

Received: September 30, 2006

Accepted: January 8, 2007

\begin{abstract}
The current research aimed to investigate the effects of early postnatal exposure to low-level lead on the spatial learning of Long-Evans Hooded rats tested in the Morris water maze. To explore possible neurotoxic actions of lead on the N-methyl-D-aspartate (NMDA) receptors, the noncompetitive NMDA receptor antagonist (+)-5-methyl-10,11-dihydroxy-5hdibenzo(a,d)cyclohepten-5,10-imine (MK-801) was used. Two-day-old pups were randomly assigned to $0.1 \%$ lead carbonate diet or control diet and weaned onto regular food on postnatal day (PND) 23. Spatial acquisition was assessed from PND 24 to PND 27. Thirty minutes prior to behavioral testing, rats received an intraperitoneal (i.p.) injection of $0.1 \%$ MK-801 or saline. In the saline treatment group, $\mathrm{Pb}$-exposed rats exhibited significantly longer overall mean escape latencies than those on the control diet, replicating the impaired spatial learning of $\mathrm{Pb}$-exposed animals tested at a young age. Although MK-801 injection severely impaired animals' water maze performance regardless of diet, it affected the performance of Pb-exposed animals to a lesser extent than animals on the control diet. No main effects of diet and drug were found for probe trials on PND 28 and PND 36, but interestingly, lead diet/MK-801 animals performed significantly better than control diet/MK-801 animals during the first probe trial. Unfortunately, MK-801 not only caused animals to display higher activity levels in the activity box, but also significantly impaired animals' performance in the cued trial, suggesting non-specific sensorimotor deficits induced by MK-801 treatment might be responsible for animals' poor performance. However, a significant drug by diet interaction in the escape latencies and the significantly better probe trial performance of $\mathrm{Pb}$-exposed animals within the MK-801 treatment group imply that lead exposure actually alleviated behavioral deficits induced by MK-801 injection, providing evidence for a possible interaction between lead and MK-801 at the molecular level.
\end{abstract}

\section{INTRODUCTION}

Since the 1970s, the detrimental effect of chronic lead exposure on cognitive performance has raised concerns in society. Many studies show that developing brains with incomplete blood-brain-barriers are most susceptible to the devastating effect of lead [1-2], and there is a high correlation between children's blood lead concentration and their intellectual performance [3-6]. Numerous correlational studies also reveal that even low-level lead exposure during early development can lead to reduced IQ, sensory deficits, associative deficits and impaired verbal performance [4-5,7-8]. Furthermore, these cognitive deficits may persist into late childhood [9-10]. Nevertheless, correlational studies, which involve no active manipulation of the variables, are inherently deficient in establishing a causal relationship between lead exposure and cognitive deficits. Unfortunately, formal controlled experiments that can better characterize the consequences of lead exposure have rarely 
been carried out so far.

However limited in numbers, previous experiments that did examine the effect of lead have shown that lead exposure causes spatial memory deficits in rodents tested in the Morris water maze [1,11]. The Morris water maze is a standard protocol assessing rodents' ability to use external cues to remember the spatial location of a submerged platform [12-13]. Although the exact mechanism by which lead impairs spatial memory is not well understood, various studies have suggested that lead exerts its neurotoxic effect by altering $\mathrm{N}$ methyl-D-aspartate (NMDA) receptors. NMDA receptors are critically involved in the production of long-term potentiation (LTP), a widely accepted cellular model for learning and memory [1, 14-17]. This makes NMDA receptors a particularly attractive site for studying lead effects.

NMDA receptors, which are highly expressed in the hippocampus, can be targeted by administering suitable antagonists. The drug (+)-5-methyl-10,11dihydroxy-5h-dibenzo(a,d)cyclohepten-5,10imine (MK-801), a non-competitive NMDA receptor antagonist that binds to the inside of the ion channel, is often used to assess the role of NMDA receptors in various learning paradigms. Previous studies have shown that systemic MK-801 administration produces impairments in acquired odor aversion, learned emotional responses, passive avoidance, spatial learning tasks, spatial working memory and reference memory in rodents [18-22]. If exposure to low-level lead results in alterations of NMDA receptors, one would expect an interaction between lead and MK-801 to manifest on the behavioral level.

The current study therefore investigates whether early postnatal exposure to lowlevel lead impairs spatial learning and memory of rats tested in the Morris water maze, and how this exposure interacts with the effect of MK-801. As some recent studies demonstrate that both lead exposure and MK-801 administration can induce hyperactivities in animals [23-24], this side effect on motor activity may confound behavioral data from the Morris water maze. Therefore, the hyperactivity associated with lead and MK-801 was controlled using an activity box in this study.

\section{METHOD}

a. Animals

Eight nursing mother rats with crossfostered 2-day old pups (Long-Evans Hooded) were obtained from Charles River (Quebec, Canada). The 80 pups were culled by gender into 10 pups per litter, and each litter was housed in a standard plastic cage with ad lib access to food (ground chow) and water. The housing room was maintained on a 12 hour dark/light cycle. Pups were randomly assigned to two diet groups. The experimental group was fed food containing $0.1 \%$ lead carbonate, and the control group with $0.1 \%$ sodium acetate. Animals were weighed every 5 days starting from postnatal day (PND) 5. They were weaned on PND 23, housed in pairs, and fed on regular rodent chow thereafter. All animal treatments were in strict accordance with the Canadian Council on Animal Care's (CCAC) Guide to the Care and Use of Experimental Animals.

\section{a. Morris Water Maze Test}

- Standard water maze test. Behavioral testing commenced on PND 24. The water maze consisted of a red plastic water tank (diameter $87 \mathrm{~cm}$ ) filled with water to a depth of $17.7-18.2 \mathrm{~cm}$. The water was maintained at room temperature $\left(20-22{ }^{\circ} \mathrm{C}\right)$, and the tank was divided into four equally sized quadrants (East, South, West, North). A transparent plastic platform was submerged $1.5-2 \mathrm{~cm}$ below the surface of the water in the East quadrant and remained in the same position throughout all tests. The water maze was located in a cued environment. Salient external cues included a video camera on a tripod (next to the Northeast quadrant) and an experimenter standing at a fixed position (next to the Southwest quadrant).

Training was performed on four consecutive days with four trials taking place each day. Thirty minutes prior to behavioral testing on each day, each rat received an i.p. injection of either $0.1 \%$ MK-801 (Tocris, Ontario, Canada) or $0.1 \%$ saline solution. The solution to be injected was randomly assigned such that approximately half of each diet group received MK-801 and the other half received saline. On the first day, 


\begin{tabular}{||l|l|l||}
\hline \multirow{2}{*}{ Age } & Diet Group & Body Weight (gm) \\
\hline \multirow{3}{*}{ PND 5 } & Control & $11.6 \pm 0.27$ \\
\cline { 2 - 3 } & Lead & $10.6 \pm 0.18^{\star}$ \\
\hline \multirow{3}{*}{ PND 10 } & Control & $21.0 \pm 0.38$ \\
\cline { 2 - 3 } & Lead & $16.8 \pm 0.41^{\star}$ \\
\cline { 2 - 3 } & Control & $33.0 \pm 0.68$ \\
\hline \multirow{3}{*}{ PND 20 } & Lead & $24.2 \pm 1.16^{\star}$ \\
\cline { 2 - 3 } & Control & $44.0 \pm 0.80$ \\
\hline \multirow{2}{*}{ PND 25 } & Coad & $35.2 \pm 1.13^{\star}$ \\
\cline { 2 - 3 } & Lead & $59.2 \pm 1.83$ \\
\hline
\end{tabular}

Table 1. Average body weight for leadexposed and control rats at 5 weighing days.

a Body weight expressed as mean + S.E.M.

* Significantly different from control rats, $p<$ .05

before the first trial, each rat received a priming trial whereby it was placed on the platform for 20 seconds. During each regular training trial, rats were dropped into a randomly assigned quadrant facing the wall of the pool. Animals were given a maximum of 60 seconds to find the platform and escape latencies were recorded by viewing a video monitor. If the platform was not found within 60 seconds, animals were placed on the platform for another 30 seconds, and the second trial only started after all rats had completed the first trial. Two replications of the experiment were performed with 24 rats in the first replication and 34 in the second (22 rats died before behavioral testing and were thus removed from the study).

- $\quad$ Probe trials. The first probe trial was performed on PND 28. Thirty minutes prior to testing, all rats received an i.p. injection of either $0.1 \%$ MK-801 or saline as previously assigned. In this trial, the platform was removed, and the amount of time animals spent in the East quadrant was recorded during a 60-second interval. A second probe trial was conducted on PND 36 and there was no injection prior to this test.
- $\quad$ Cued trial. Following the probe trial on PND 28, a cued trial was performed. In this trial the platform was submerged in the North quadrant and a flag was placed over the platform. Each rat was again given a maximum of 60 seconds to find the platform and escape latency was recorded.

\section{Activity Box}

After the cued trial was completed on PND28, all rats were individually evaluated on their motor activities in an activity box (base area $80 \mathrm{~cm} \times 80 \mathrm{~cm}$, height $32.45 \mathrm{~cm}$ ), the bottom of which was marked by a grid of 25 equally sized squares $(15 \mathrm{~cm} \times 15 \mathrm{~cm})$. Rats were placed onto the center square and their activity levels were measured in three 5-minute intervals. Activity was measured by counting the number of times the head and front two paws cross over one gridline or walk diagonally over an intercept. The testing box was cleaned following each 15-minute session.

\section{d. Statistical Analysis}

All data were analyzed using SPSS 14.0. One-way analysis of variance (ANOVA) was performed on body weights and probe trial 2 performance. Overall mean escape latency, probe trial 1 performance, cued trial escape latency and activity levels were analyzed using $2 \times 2$ ANOVA, treating diet and drug as between-subject factors. Correlations between overall mean escape latency and other relevant data (cued trial escape latency and activity levels) were also calculated. All statistics were considered significant when $p<.05$.

\section{RESULTS}

\section{a. Weight}

Animals were weighed every five days starting from PND 5 (Table 1). Pb-exposed rats weighed significantly less than control rats on the following weighing days: PND 5 $[F(1,60)=8.505, p<.05]$, PND $10[F(1,57)$ $=58.076, p<.05]$, PND $15[F(1,57)=$ 44.013, $\mathrm{p}<.05]$ and PND $20[\mathrm{~F}(1,56)=$ 40.562, $p<.05]$. On PND 25, which was 2 days after weaning, there was no longer a significant difference in body weight between the two diet groups $[F(1,56)=$ $0.198, p=.658]$. 


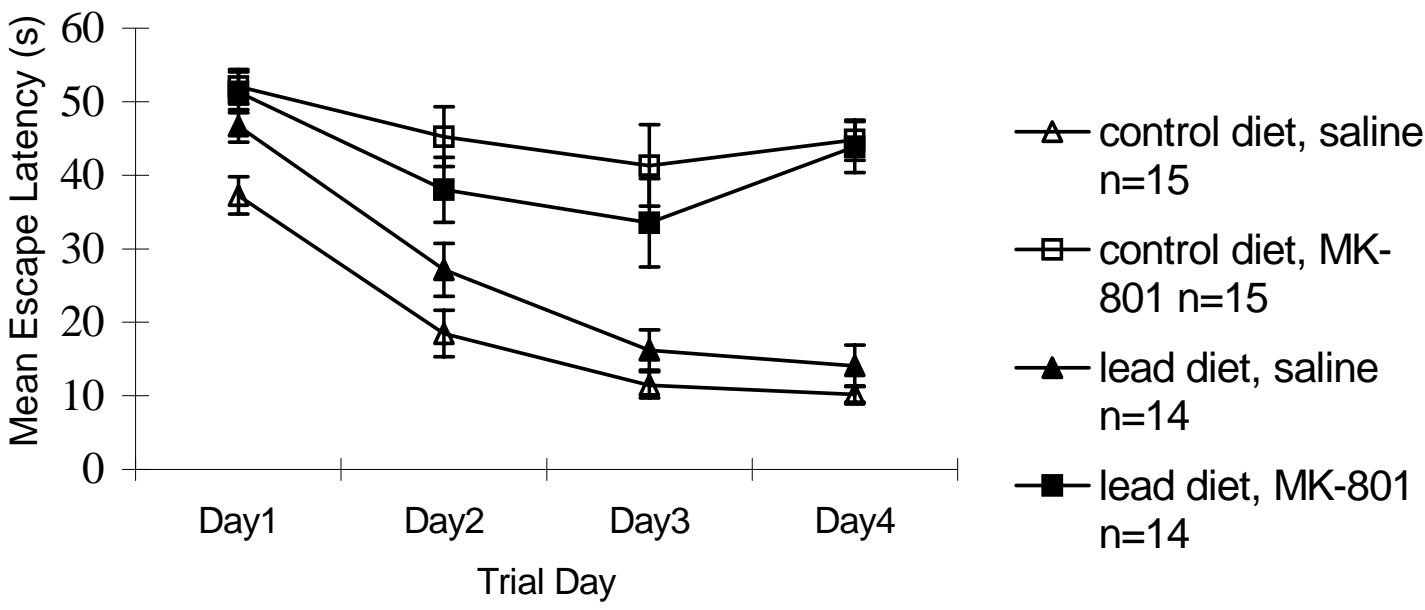

Figure 1. Mean escape latency at each trial day for each treatment group (mean + S.E.M).

\section{b. Morris Water Maze performance}

- $\quad$ Standard water maze test. The trend of daily mean escape latencies for all treatment groups was shown in Figure 1. As can be seen in this figure, all animals regardless of diet or drug pretreatment showed decreased escape latency over days, indicating learning had taken place.

One-way repeated measure analysis showed that all four groups displayed significant or nearly significant savings on learning across the four training days (control diet/saline $[F(3,42)=43.369, p<$ .01]; control diet/MK-801 $[F(3,42)=2.823, p$ $=.0502]$; lead diet $/$ saline $[F(3,39)=40.107$, $p<.01]$; lead diet/MK-801 $[F(3,39)=5.702$, $p<.01])$. The overall mean escape latency (average of all 16 trials) was computed for each rat. The ANOVA revealed a significant main effect of drug $[F(1,54)=70.483, p<$ $.01]$, with MK-801-treated rats displaying significantly longer overall escape latency $(43.70 \pm 2.119 \mathrm{~s} ; \mathrm{n}=29)$ than saline treated rats $(22.58 \pm 1.439 \mathrm{~s} ; \mathrm{n}=29)$. This significant difference was consistent throughout four trial days. Although there was no significant main effect of diet on overall escape latency, a significant simple effect of diet was revealed when data analysis was performed on results from saline treated rats only $[F(1,28)=6.411, p<$ .05]. Among rats that were injected with saline prior to behavioral testing, $\mathrm{Pb}$ exposed rats took a significantly longer time $(26.03 \pm 2.134 \mathrm{~s} ; \mathrm{n}=14)$ than controls $(19.35 \pm 1.588 \mathrm{~s} ; \mathrm{n}=15)$ to find the hidden platform (Figure 2). Further analysis revealed a significant difference in the escape latency between the two groups on the first day of training $[F(1,27)=7.75, p=$ .010] even though the difference fell to a non-significant level starting from training day 2 .

Interestingly, the data analysis indicated a significant drug by diet interaction $[F(1,54)$ $=4.954, p<.05]$, implying differential effects of lead exposure on the two injection groups. While $\mathrm{Pb}$-exposed animals showed significantly worse performance than animals on the control diet within salinetreated group, among MK-801-treated rats, $\mathrm{Pb}$-exposed rats even displayed slightly shorter escape latencies (41.41 $\pm 3.273 \mathrm{~s}$; $\mathrm{n}$ $=14)$ than those on control diet $(45.84 \pm$ $2.720 \mathrm{~s} ; \mathrm{n}=15$ ) even though the difference was not statistically significant $[F(2,37)=$ 1.09, $p=.305$ ] (Figure 2). It was surprising that lead exposure did not exacerbate behavioral deficits induced by MK-801 injection, and even exhibited a tendency to alleviate the degree of impairment caused by MK-801 injection from baseline condition (saline injection). 


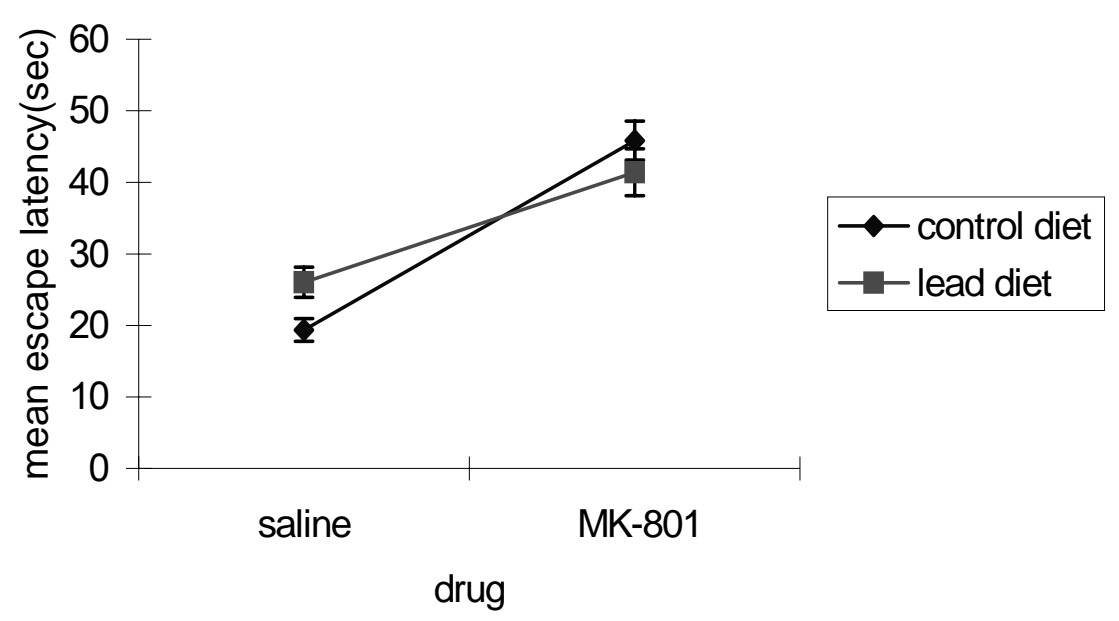

Figure 2. Overall mean escape latency for all treatment groups (mean + S.E.M). ** Significantly different from saline treated rats, $p<.01$; * Significantly different from saline treated rats on control diet, $\mathrm{p}<.05$

- $\quad$ Probe trials. Probe trials measured the amount of time rats spent in the East quadrant, the quadrant in which the hidden platform was previously located. There was no significant main effect of either diet or drug on both probe trials. Nevertheless, a significant simple effect of diet was revealed when considering probe trial 1 performance among MK-801-treated rats only $[\mathrm{F}(1,27)=$ $5.20, \mathrm{p}<.05]$. Control diet/MK-801 rats spent significantly less time $(11.27 \pm 1.123$ $\mathrm{s} ; \mathrm{n}=15$ ) in the target quadrant than lead diet/MK-801 rats $(15.86 \pm 1.703 \mathrm{~s} ; \mathrm{n}=14)$ (Figure 3).

- $\quad$ Cued trial. A strong correlation was found between cued trial escape latency and mean escape latency $(r=0.721, p<.01)$ (Figure 4). Also, there was a significant main effect of drug on animals' performance in this visual platform trial $[F(1,54)=44.311, p$ $<$.01]. MK-801-treated rats took a significantly longer time $(35.62 \pm 4.043 \mathrm{~s} ; \mathrm{n}$ $=29)$ than saline treated rats $(8.41 \pm 0.685$ $\mathrm{s} ; \mathrm{n}=29)$ to find the platform. Moreover, $31 \%(n=9)$ of MK-801-treated rats were unable to find the platform within 60-second time limit, suggesting that severe visual deficits had been caused by the drug administration. There was no main effect of diet found in this trial. Neither did any drug by diet interaction exist.

\section{c. Activity Box}

Since the activity levels at each 5minute interval were significantly correlated with the total activity levels (first interval: $r=$ 0.857, $p<.01$; second interval: $r=0.774, p$ $<.01$; third interval: $r=0.728, p<.01$ ), only the total activity levels were analyzed. The ANOVA indicated a significant main effect of drug on animals' activity levels $[F(1,54)=$ 23.667, $p<.01$ ] (Figure 5).

Rats injected with MK-801 displayed significantly greater activity levels than those injected with saline. There was neither a main effect of diet nor any drug by diet interaction found. Animals' activity levels were also uncorrelated with the overall mean escape latency.

\section{DISCUSSION}

The present study shows that early postnatal exposure to low-level lead impaired the performance of drug-free animals in the Morris water maze, but not MK-801-treated animals. Among saline treated rats, the ones exposed to the lead diet exhibited significantly longer overall mean escape latency than those on the control diet. Although MK-801-treated rats were all severely impaired during training, $\mathrm{Pb}$-exposed ones showed comparable, if not better, performance as compared to those 


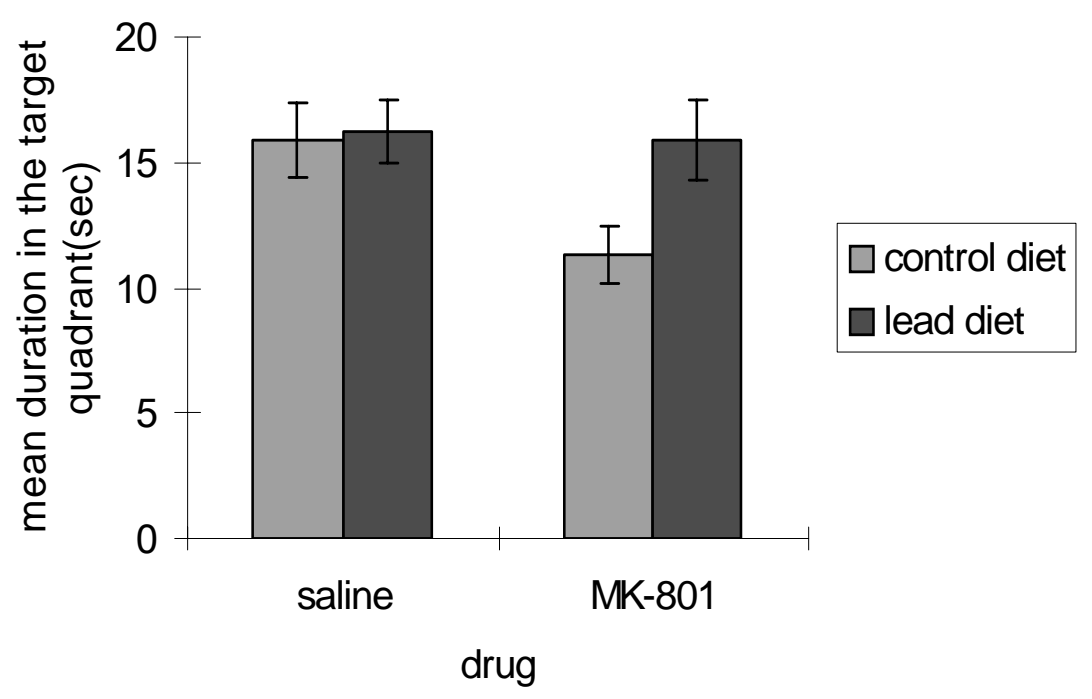

Figure 3a. Mean duration in the target quadrant for all treatment groups (control diet/saline, lead diet/saline, control diet/MK-801, lead diet/MK-801) during probe trial 1, shown as mean + S.E.M. * Significantly different from lead diet/MK-801 rats, $p<.05$

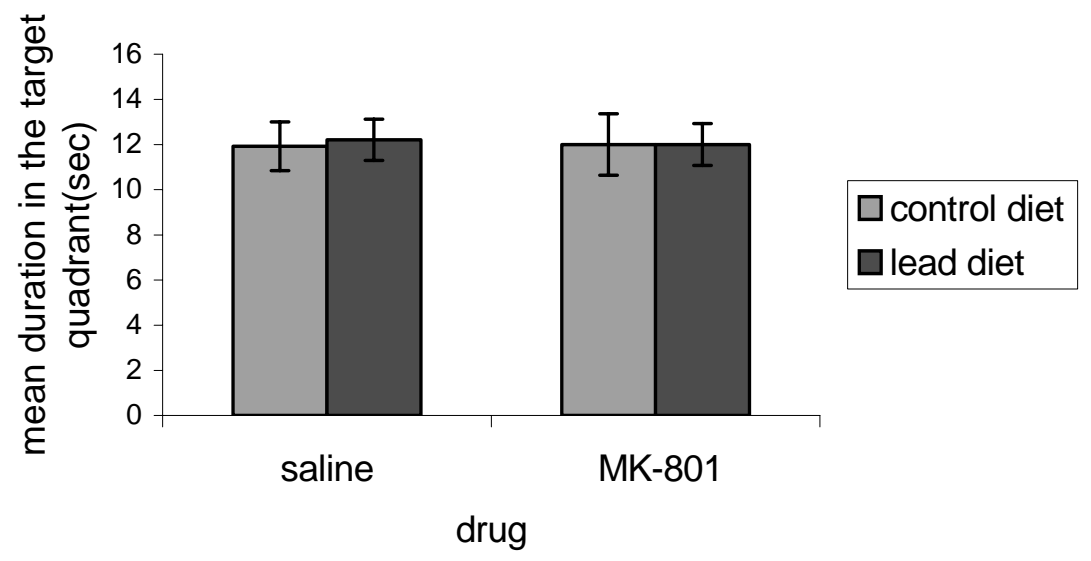

Figure 3b. Mean duration in the target quadrant for all treatment groups (control diet/saline, lead diet/saline, control diet/MK-801, lead diet/MK-801) during probe trial 2, shown as mean \pm S.E.M.

on the control diet. More importantly, lead diet/MK-801 animals performed significantly better than control diet/MK-801 animals in probe trial 1, further suggesting a possible alleviation of MK-801 induced deficits as a result of low-level lead exposure.

Together with previous studies [2], the findings suggest that both prenatal and early postnatal exposure to low-level lead affect animals' spatial learning ability tested at a young age (between PND 20 and PND 30). In Jett et al.'s experiment, lead-exposed animals that started behavioral testing on PND 21 were also impaired in the probe trial. In this current study, however, $\mathrm{Pb}$ exposed animals performed equally well with control animals in both probe trials, suggesting that low-level lead only slows down the acquisition of spatial memory without affecting its consolidation and retrieval. These inconsistent results might be obtained due to differences in the starting time and route of lead administration. In this experiment, instead of implementing 


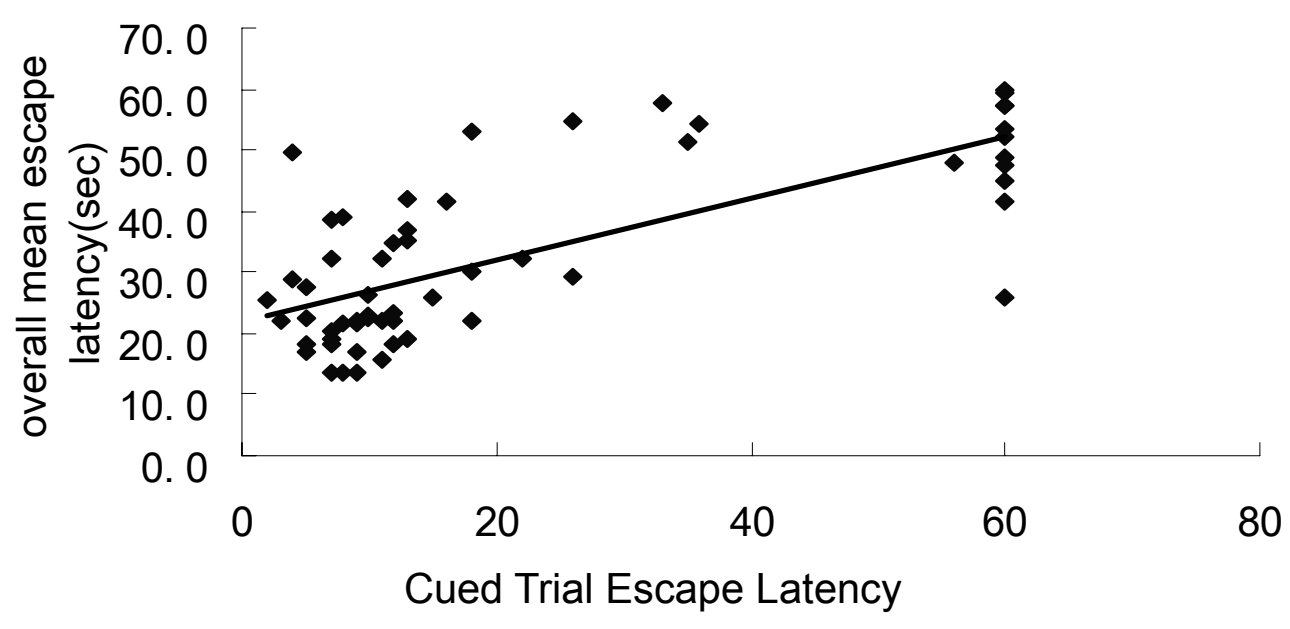

Figure 4. Correlation between cued trial escape latency and overall mean escape latency during training sessions $(r=0.721, p<.01)$.

prenatal exposure through drinking water, pups were exposed to $\mathrm{Pb}$-containing food when they were 2 days old. It is possible that the concentration of lead accumulated in the $\mathrm{Pb}$-exposed animals' hippocampi was insufficient to impair the formation and stabilization of long-term spatial memory. Further studies are needed to elucidate the exact relationship between hippocampal lead concentration and its cognitive consequence on different components of learning and memory, including acquisition, consolidation and retrieval.

It could be argued that since $\mathrm{Pb}$ exposed animals performed equally well with controls in both probe trials, any observed differences in the escape latency during training sessions should not be attributed to learning deficits; instead they are likely to be caused by more general effects of the chemical. However, several reasons may explain why this is not the case. First of all, non-specific effects of lead were well controlled in this experiment. The weighing data did show that animals on the lead diet were significantly lighter than those on the control diet up to PND 20. However, on PND 25, there was no longer a significant difference between the two diet groups. Considering the fact that behavioral testing was conducted between PND 24 and 27, it is more reasonable to assume that weight is not a contributing factor to animals' escape latency. Also, among MK-801-treated rats, those on lead diet performed even slightly better than the controls. Therefore, factors other than physical weight should account for the observed behavioral differences. Activity levels of animals were also controlled in this study. In contrast to what previous studies suggest, lead exposure in the present study did not lead to hyperactivity in animals [24]. In fact, $\mathrm{Pb}$ exposed animals exhibited similar activity levels with control animals. This may be due to the lower concentration of lead used in this experiment. Moreover, lead diet had no impairing effect on animals' cued trial performance. Since $\mathrm{Pb}$-exposed animals showed longer escape latency only in the absence but not in the presence of cue, slower learning caused by lead exposure should not be attributed to either visual problems or lower motivation level. After excluding the several non-cognitive effects that may possibly influence the performance of $\mathrm{Pb}$-exposed animals, it is reasonable to assume that any behavioral impairment is probably an outcome of cognitive deficits associated with lead exposure. Secondly, it should be emphasized that although spatial learning/memory appears to be one unified phenomenon, it actually consists of a few distinct phases, namely the acquisition, consolidation and retrieval. Impairment could possibly occur at one single phase without affecting other phases. It is true that $\mathrm{Pb}$-exposed animals eventually acquired as 


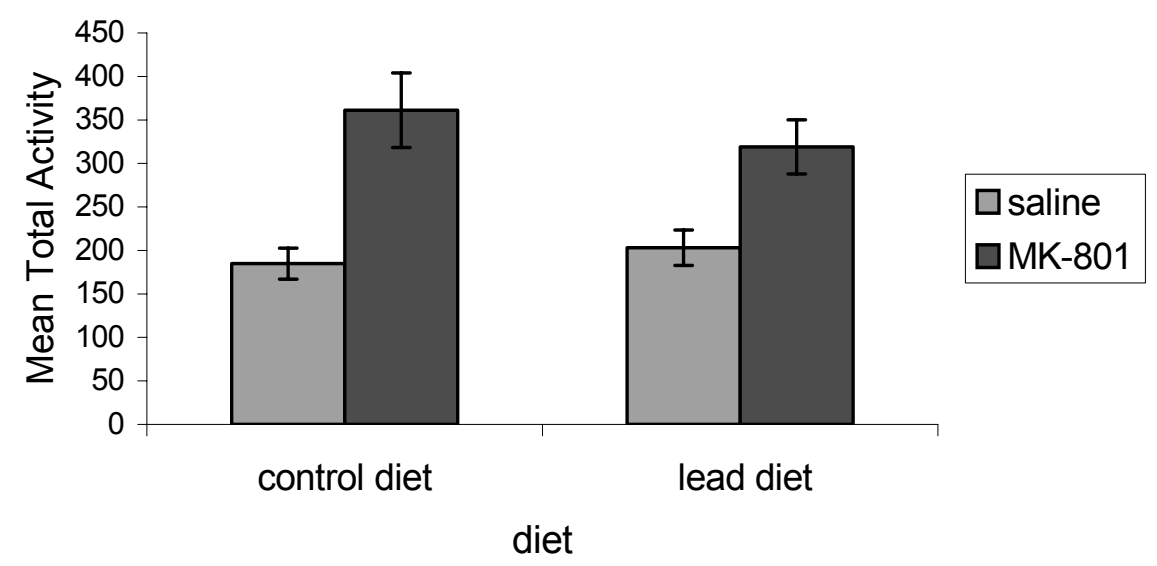

Figure 5. Mean total activity levels for all treatment groups (control diet/saline, lead diet/saline, control diet/MK-801, lead diet/MK-801), shown as mean \pm S.E.M. ${ }^{* *}$ Significantly different from saline treated rats, $p<.01$

robust a memory as the control animals, but a significant difference in the escape latency on the first training day and a significantly prolonged overall mean escape latency undeniably pointed to an observable deficit in the acquisition of spatial memory, no matter how small the deficit is and how closely the overall trend in daily escape latency matched with the controls.

Since NMDA receptors are critically involved in learning and memory, they are proposed to be the target site where lead exerts its neurotoxic effect $[1,14-15]$. The NMDA receptor antagonist MK-801 was therefore used in the current study to probe for the possible mechanisms of lead action. Consistent with previous experiments, the administration of MK-801 seemed to result in significant impairment of animal's spatial learning [19-20]. One may attribute the impairment to the significantly higher activity levels induced by MK-801 injection. However, since MK-801-treated rats spent a significantly longer time to find the hidden platform, hyperactivity, which tends to increase the probability of finding the platform by chance, would not account for their poorer performance. Moreover, there was no correlation between the activity levels and water maze performance. Unfortunately, MK-801-treated rats showed significantly longer escape latency than saline treated rats in the cued trial as well, suggesting severe visual problems caused by the drug. The data analysis also illustrates a significant correlation between cued trial escape latency and normal trial escape latency, implying that animals which had longer escape latencies during normal trials may not be cognitively impaired after all, and perhaps it is their visual problem that affected their performance. This interpretation should be taken seriously especially owing to the fact that $31 \%$ of MK801 -treated rats while $0 \%$ of saline-treated rats were unable to find the visible platform within $60 \mathrm{~s}$. Therefore, one should not be making blunt claims that MK-801 impairs spatial learning and memory, because in the current study, the effect of MK-801 on higher-order cognitive processing, such as the ability to associate external cues with the platform location, was masked by perceptual deficits. In other words, since the behavioral test heavily relies on animals' visual system to pick up external cues, it does not allow for a fair judgment on animals' ability to learn and memorize when some of them developed poor vision. It is worth mentioning that factors other than visual problems, such as altered motivational level, may also contribute to a general increase in the escape latency during both cued and normal trials.

Provided both lead and MK-801 have an impairing effect on water maze performance regardless of the actual nature of impairment, one might expect animals that are both $\mathrm{Pb}$-exposed and $\mathrm{MK}-801-$ treated to perform the worst. Surprisingly, instead of exacerbating the deficits caused by MK-801, lead tended to alleviate the 
effect of MK-801. Although MK-801-treated rats did not significantly differ between the two diet groups during normal training sessions, a significant drug by diet interaction demonstrates that MK-801 injection did affect $\mathrm{Pb}$-exposed animal to a significantly lesser degree. One possible explanation which could not be dismissed is that there might be a ceiling effect among the MK-801-treated rats, so that further deficits were prevented from showing up in the data, and the slightly better performance of $\mathrm{Pb}$-exposed animals was simply due to sampling errors. More sensitive measures, such as the direction of initial heading and the total path length, may be used in future studies to distinguish the performance of the two diet groups under MK-801 treatment. Nevertheless, a significant better performance of $\mathrm{Pb}$-exposed animals within the MK-801 treatment group in probe trial 1 strongly suggests that lead does abate the deleterious effect of MK-801 to a measurable degree. No such difference showed up in probe trial 2 however, because all animals were tested under a drug-free condition. This suggests that any deficits caused by MK-801 do not have a prolonged effect.

Admittedly, since it is not entirely clear what actually caused the worse performance of MK-801-treated animals, it is difficult to determine whether lead actually has a mild protection effect on learning and memory in the special circumstance of MK-801 treatment or it simply tempers non-cognitive deficits induced by MK-801. Given that nonspecific effects of MK-801 offer probable explanations for the observed behavioral deficits, this study provides no evidence of hippocampal involvement in the MK-801 induced deficits. Nevertheless, some kind of interaction between lead and MK-801 must have taken place somewhere in the brain if lead does alleviate the deleterious effects of MK-801. As discussed above, the observed deficits induced by MK-801 injection may likely due to visual problems, thus the interaction between lead and MK-801 could conceivably have taken place in the visual cortex of rats. Furthermore, what is unsure is just where in the brain MK-801 exerts an impairing effect but not how it exerts its effects, because any consequence of MK801 administration always arises from it noncompetitive binding to the NMDA receptors.
Consequently, the possibility of non-specific effects of MK-801 should not deter one from making reasonable hypothesis that the key to the lead-MK-801 interaction lies at NMDA receptors. Previous studies failed to reach an agreement on whether lead increases or decreases NMDA receptor density $[1,15-17]$. Data from the present study would suggest that exposure to low-level lead more likely causes an increase in the NMDA receptor density at the affected brain region. The reasoning is as follows: a given dosage of MK-801 would affect a specific number of NMDA receptors; if the number of NMDA receptors now increases, due to the existence of more functional NMDA receptors, the same dosage of MK-801 would not cause as much impairment as before. This is exactly what the results demonstrated. However, this proposed molecular mechanism of lead action is highly speculative, because the existence of an interaction between lead and MK-801 is itself not conclusive from the present study.

If this speculation is true, one may then question why $\mathrm{Pb}$-exposed animals showed slower acquisition than the controls under saline condition even though they have raised NMDA receptors density. A reasonable explanation is that although NMDA receptors play critical roles in learning and memory, more NMDA receptors do not always correspond to enhanced learning and memory. In fact, this explanation is supported by previous studies which demonstrated that most efficient learning requires an optimal instead of a maximum level of NMDA receptors ([25] Ingram et al., 1992; [26] Brooks et al., 1997). Perhaps in $\mathrm{Pb}$-exposed animals, excess receptors disrupted normal synaptic plasticity, thus leading to impaired spatial learning.

In conclusion, the present study shows that early postnatal exposure to even lowlevel lead can lead to observable impairment in the acquisition of spatial memory, and a $\mathrm{Pb}$-induced alleviation of MK-801 associated deficits provides evidence for a possible alteration of NMDA receptor density by lead. Further experiments are necessary to verify the existence of an interaction between lead and MK-801 at the molecular level and also to explore the effects of low-level lead on other cognitive capacities. 


\section{ACKNOWLEDGEMENTS}

This research was supported by University of Toronto at Scarborough Neuroscience Program funding. I would also like to thank my NROC63 course classmates for their immense contributions to this project, and thank Dr. LeBoutillier, Tayyeba and Michelle for their invaluable guidance.

\section{REFERENCES}

1. Jett DA, Guilarte TR. "Developmental lead exposure alters $N$-methyl-Daspartate and muscarinic cholinergic receptors in the rat hippocampus: an autoradiographic study," Neurotoxicology 16 (1995) 7-18.

2. Jett DA, Kuhlmann AC, Farmer SJ, Guilarte TR. "Age-dependent effects of developmental lead exposure on performance in the Morris water maze," Pharmacol. Biochem. Behav. 57(1-2) (1997) 271-279.

3. Ruff HA, Markowitz ME, Bijur PE, Rosen JF. "Relationships among blood lead levels, iron deficiency, and cognitive development in two-year-old children," Environ. Health Perspect. 104 (1996) 180-185.

4. Canfield RL, Kreher DA, Cornwell C, Henderson CR Jr. "Low-level lead exposure, executive functioning, and learning in early childhood," Child Neuropsychol. 9(1) (2003) 35-53.

5. Coscia JM, Ris MD, Succop PA, Dietrich KN. "Cognitive Development of Lead Exposed Children from Ages 6 to 15 Years: An Application of Growth Curve Analysis," Child Neuropsychol. 9(1) (2003) 10-21.

6. Wasserman GA, Factor-Litvak P, Liu $\mathrm{XH}$, Todd AC, Kline JK, Slavkovich V, Popovac D, Graziano JH. "The Relationship Between Blood Lead, Bone Lead and Child Intelligence," Child Neuropsychol. 9(1) (2003) 22-34.

7. Lanphear BP, Dietrich K, Auinger P, Cox C. "Cognitive Deficits Associated with Blood Lead Concentrations [less than] $10 \mu \mathrm{g} / \mathrm{dL}$ in US Children and Adolescents," Public Health Reports 115(6) (2000) 521.

8. Canfield RL, Henderson CR Jr, CorySlechta DA, Cox C, Jusko TA, Lanphear BP. "Intellectual impairment in children with blood lead concentrations below 10 microg per deciliter," N. Engl. J. Med. 348(16) (2003) 1517-1526.

9. Baghurst PA, McMichael AJ, Wigg NR, Vimpani GV, Robertson EF, Roberts RJ, Tong SL. "Environmental exposure to lead and children's intelligence at the age of seven years. The Port Pirie Cohort Study," N. Engl. J. Med. 327(18) (1992) 1279-1284.

10. Bellinger DC, Stiles KM, Needleman HL. "Low-level lead exposure, intelligence and academic achievement: a long-term follow-up study," Pediatrics 90(6) (1992) 855861.

11. Jett DA, Kuhlmann AC, Guilarte TR. "Intrahippocampal administration of lead $(\mathrm{Pb})$ impairs performance of rats in the Morris water maze," Pharmacol. Biochem. Behav. 57(1-2) (1997) 263269.

12. Morris RG, Garrud P, Rawlins JN, O'Keefe J. "Place navigation impaired in rats with hippocampal lesions," Nature 297 (1982) 681-683.

13. Young GS, Choleris E, Kirkland JB. "Use of salient and non-salient visuospatial cues by rats in the Morris Water Maze," Physiol Behav. 87(4) (2006) 794-799.

14. Guilarte TR, Miceli RC, Altmann L, Weinsberg F, Winneke G, Wiegand $H$. "Chronic prenatal and postnatal $\mathrm{Pb2+}$ exposure increases [3H]MK801 binding sites in adult rat forebrain," Eur. J. Pharmacol. 248(3) (1993) 273275.

15. Lasley SM, Polan-Curtain J, Armstrong DL. "Chronic exposure to environmental levels of lead impairs in vivo induction of long-term potentiation in rat hippocampal dentate," Brain Res. 614 (1993) 347-351.

16. Ma T, Chen HH, Lim DK, Hume AS, Ho IK. "Effects of subacute lead exposure on $[3 \mathrm{H}] \mathrm{MK}-801$ binding in hippocampus and cerebral cortex in the adult rat," Brain Res. 760(1-2) (1997) 187-192.

17. Lasley SM, Green MC, Gilbert ME. "Rat hippocampal NMDA receptor binding as a function of chronic lead exposure level," Neurotoxicol. Teratol. 23(2) (2001) 185-189. 
18. Mondadori C, Weiskrantz L, Buerki H, Petschke F, Fagg GE, "NMDA receptor antagonists can enhance or impair learning performance in animals," Exp. Brain Research 75 (1989) 449-456.

19. Robinson GS, Crooks GB Jr, Shinkman PG, Gallagher M. "Behavioral effects of MK-801 mimic deficits associated with hippocampal damage," Psychobiology 17 (1989) 156-164.

20. Shapiro ML, Caramanos Z. "NMDA antagonist MK-801 impairs acquisition but not performance of spatial working and reference memory," Psychobiology 18 (1990) 231-243.

21. Hoehn-Saric R, McLeod DR, Glowa JR. "The effects of NMDA receptor blockade on the acquisition of a conditioned emotional response," Biol. Psychiatry 30 (1991) 170-176.

22. Packard MG, Teather LA. "Posttraining injections of MK-801 produce a timedependent impairment of memory in two water maze tasks," Neurobiol. Learn. Mem. 68(1) (1997) 42-50.
23. Zuo DY, Zhang YH, Cao Y, Wu CF, Tanaka M, Wu YL. "Effect of acute and chronic MK-801 administration on extracellular glutamate and ascorbic acid release in the prefrontal cortex of freely moving mice on line with openfield behavior," Life Sci. 78(19) (2005) 2172-2178.

24. Punzo F, Farmer C. "Effects of lead on exploratory behavior and running speed in the shrew, Blarina brevicauda (Insectivora)," J. Environ. Biol. 24(4) (2003) 411-414.

25. Ingram DK, Garofalo P, Spangler EL, Mantione CR, Odano I, London ED. "Reduced density of NMDA receptors and increased sensitivity to dizocilpineinduced learning impairment in aged rats," Brain Res. 580(1-2) (1992) 273280.

26. Brooks WJ, Weeks AC, Leboutillier JC, Petit TL. "Altered NMDA sensitivity and learning following chronic developmental NMDA antagonism," Physiol. Behav. 62(5) (1997) 955-962.

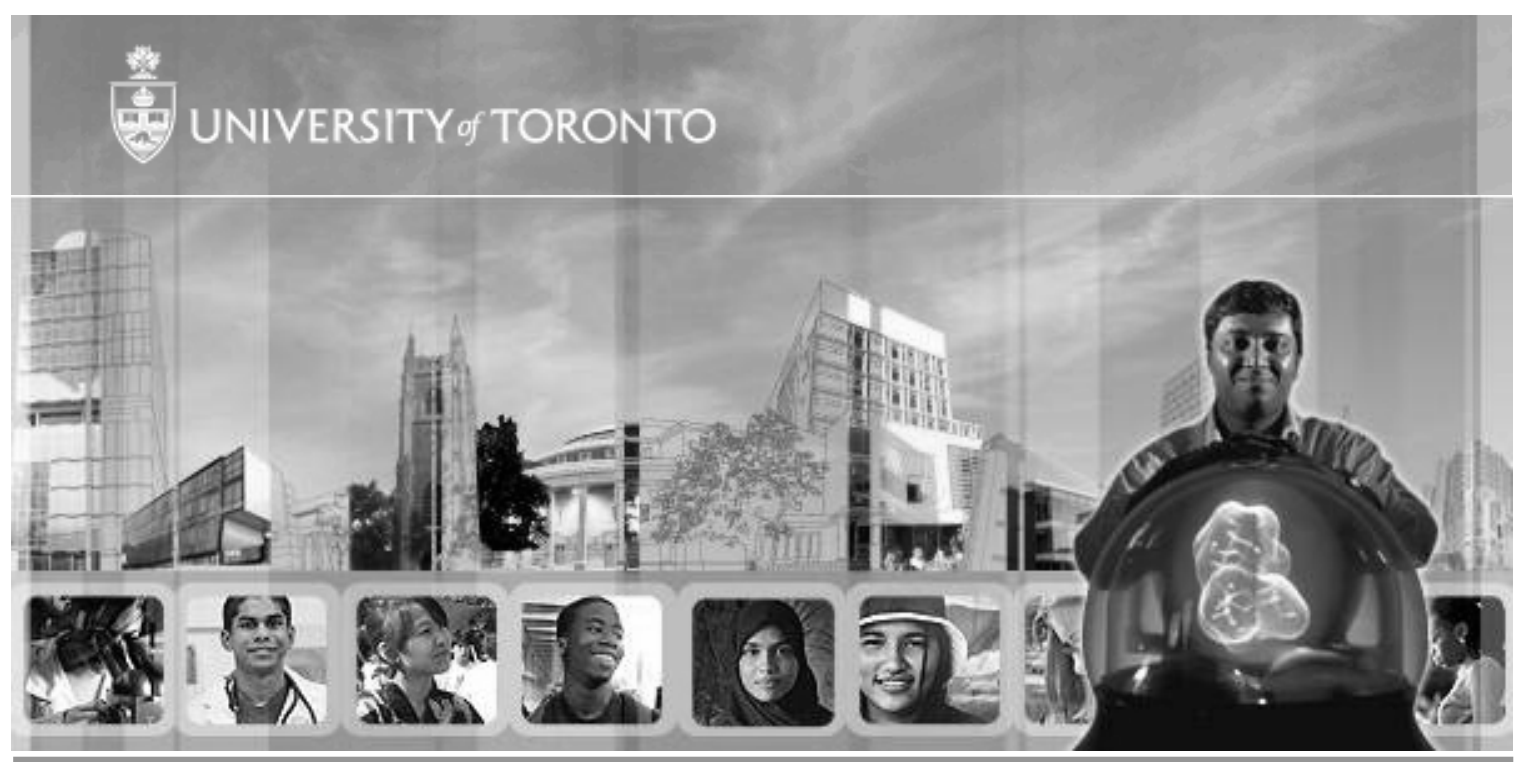

Founded in 1827, the University of Toronto is very much like the Greater Toronto Area: it is a large, multicultural community with a great number of opportunities and resources; and it is organized around neighbourhoods, where smaller gatherings of people come together to learn and think and work. In our knowledge-based age, higher education is becoming increasingly important to our prosperity and our quality of life. Working with members of our various local, national and international communities, $U$ of $\mathrm{T}$ strives to make far-reaching and positive differences in the world - through the education of our students and through our pioneering research. 


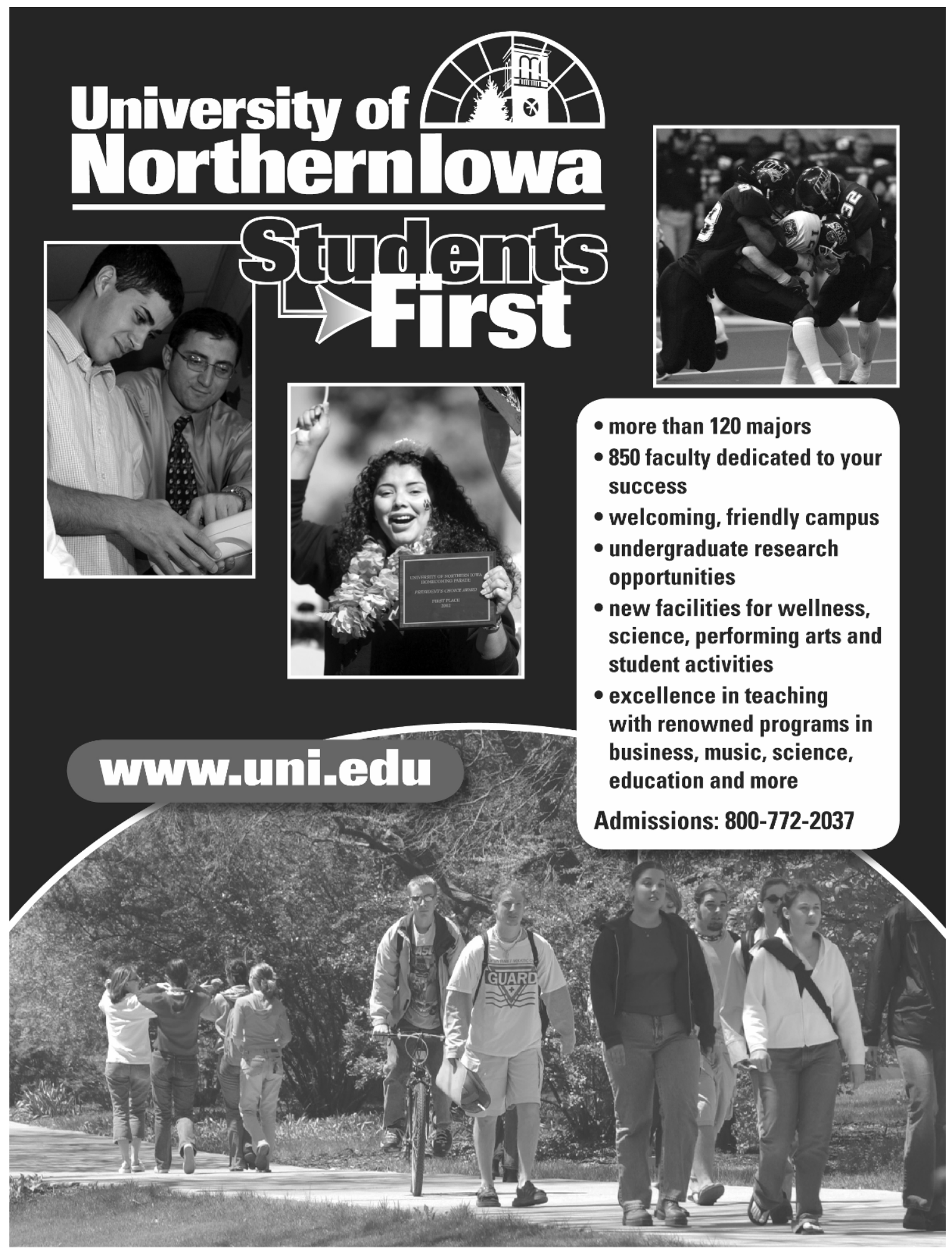

